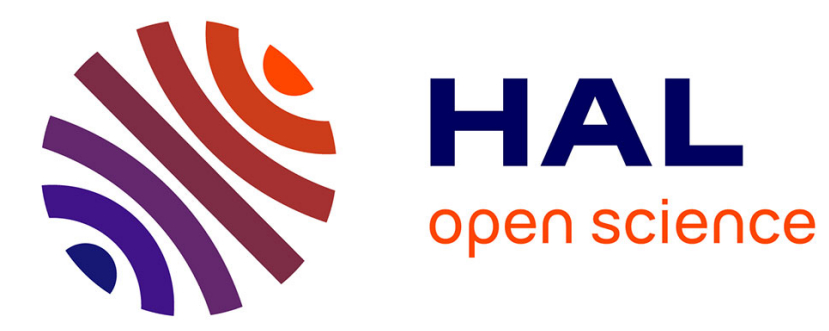

\title{
Reconstructing the formation history of top-shaped asteroids from the surface boulder distribution
}

Bin Cheng, Yang Yu, Erik Asphaug, Patrick Michel, Derek Richardson, Masatoshi Hirabayashi, Makoto Yoshikawa, Hexi Baoyin

\section{- To cite this version:}

Bin Cheng, Yang Yu, Erik Asphaug, Patrick Michel, Derek Richardson, et al.. Reconstructing the formation history of top-shaped asteroids from the surface boulder distribution. Nature Astronomy, 2020, 10.1038/s41550-020-01226-7 . hal-02986154

\section{HAL Id: hal-02986154 \\ https://hal.science/hal-02986154}

Submitted on 28 Dec 2020

HAL is a multi-disciplinary open access archive for the deposit and dissemination of scientific research documents, whether they are published or not. The documents may come from teaching and research institutions in France or abroad, or from public or private research centers.
L'archive ouverte pluridisciplinaire HAL, est destinée au dépôt et à la diffusion de documents scientifiques de niveau recherche, publiés ou non, émanant des établissements d'enseignement et de recherche français ou étrangers, des laboratoires publics ou privés. 


\section{Reconstructing the formation history of top-shaped aster- oids by the surface boulders distribution}

3 Bin Cheng ${ }^{1,3}$, Yang $\mathrm{Yu}^{2, *}$, Erik Asphaug ${ }^{3}$, Patrick Michel ${ }^{4}$, Derek C. Richardson ${ }^{5}$, Masatoshi

4 Hirabayashi $^{6}$, Makoto Yoshikawa ${ }^{7,8} \&$ Hexi Baoyin $^{1,+}$

${ }_{5}{ }^{1}$ School of Aerospace Engineering, Tsinghua University, Beijing, China.

${ }_{6} \quad{ }^{2}$ School of Aeronautic Science and Engineering, Beihang University, Beijing, China.

$7 \quad{ }^{3}$ Lunar and Planetary Laboratory, University of Arizona, Tucson, AZ, USA.

${ }_{8}^{4}$ Université Côte d'Azur, Observatoire de la Côte d'Azur, CNRS, Laboratoire Lagrange, Nice,

9 France.

${ }_{10}^{5}$ Department of Astronomy, University of Maryland, College Park, MD, USA.

${ }_{11}{ }^{6}$ Department of Aerospace Engineering, Auburn University, Auburn, AL, USA.

$12{ }^{7}$ Institute of Space and Astronautical Science (ISAS), Japan Aerospace Exploration Agency

13 (JAXA), Sagamihara, Japan.

${ }_{14}^{8}$ The Graduate University for Advanced Studies (SOKENDAI), Kanagawa, Japan.

15 *yuyang.thu@gmail.com

16 +baoyin@tsinghua.edu.cn

Finding the basic mechanism governing the surface history of asteroids of various shapes is essential for understanding their origin and evolution. In particular, the currently visited asteroids (162173) Ryugu ${ }^{1}$ and (101955) Bennu' ${ }^{2}$ by Hayabusa2 and OSIRIS-REx appear to be top-shaped. This distinctive shape, characterized by a raised equatorial bulge, is shared 
by other similarly sized asteroids, including Didymos $\mathrm{A}^{3}, 2008$ EV5 $^{4}$ and $1999 \mathrm{KW} 4$ Alpha ${ }^{5}$. However, the possibly common formation mechanism that causes the top-like shape is still under debate. One clue may lie in the boulders on their surfaces. The distribution of these boulders, that was precisely measured in unprecedented detail by the two spacecrafts ${ }^{1,2}$, constitutes a record of the geological evolution of the surface regolith since the origin of these asteroids. Here, we show that during the regolith migration driven by YORP spin-up ${ }^{6-9}$, the surface boulders coevolve with the underlying regolith and exhibit diverse dynamical behaviors: they can remain undisturbed, sink into the regolith layer and become tilted, or totally be buried by the downslope deposition, depending on their latitudes. The predominant geological features commonly observed on top-shaped asteroids, including the boulder-rich region near the pole ${ }^{1,10}$, the deficiency of large boulders at the equatorial area ${ }^{10,11}$ and partially buried, oblique boulders exposed on the regolith surface ${ }^{12,13}$, are commensurate with this coevolution scenario. The surface regolith migration thus is the prevalent mechanism for the formation history of the top-shaped asteroids with stiffer cores.

Our investigation of the quasi-static process of boulder-regolith evolution by YORP spinup was performed using numerical code based on the soft-sphere discrete element method ${ }^{14-16}$ (SSDEM). The considered structure and surface of a top-shaped asteroid consist of a "two-layer structure" with a shallow mantle of loose granular regolith covering a stiffer interior ${ }^{7,8}$. This model is supported by multiple observations of the surface geologic features on Ryugu and Bennu, e.g., the longitudinal ridges and long linear grooves denoting internal stiffness and structural rigidity with a weak strength of less than a few $\mathrm{Pa}^{17}$; the measured thermal inertia and substantial material 
flows evidencing loose and unconsolidated surface regolith ${ }^{1,13}$. The pit feature of the artificial impact crater on Ryugu provides further evidence of a stronger substrate covered by cohesionless regolith ${ }^{18}$. This heterogeneity could appear as a result of stronger interparticle cohesion, grains of different materials, higher packing fraction, a big inner monolith, etc., or a combination of those particular characteristics ${ }^{8}$. The "granular shell" model is shown in Fig. 1a, i.e., the asteroid interior is modelled as a rigid spheroid ( $\sim 400 \mathrm{~m}$ in radius), and a longitudinal slice of the regolith layer ( $\sim 30 \mathrm{~m}$ in thickness) is considered for the SSDEM simulation, consisting of 2,063,044 pebble-size particles. The boulders of various sizes, modelled as massive polyhedra, are located at four latitudes $\left\{15^{\circ}, 45^{\circ}, 75^{\circ}, 87^{\circ}\right\}$ as representatives of low, mid-low, mid-high, and high latitudes, respectively. We assumed these boulders are initially on top of the regolith layer, which could be the direct result of the re-accumulation that formed the asteroids after the catastrophic disruption of their parent bodies ${ }^{12,13}$, or much later by ballistic sorting ${ }^{19}$ or Brazil-nut effect ${ }^{20}$ during impactinduced seismic events. Two different types of regolith grains were used: one with moderate friction (internal friction angle of $\sim 25^{\circ}$ ) and one with gravel-like friction (internal friction angle of $\sim 30^{\circ}$ ). We then used two types of regolith grains for each of the four latitudes, and each of the three boulders, giving a total of 24 simulations (all simulation parameters are summarized in the supplementary information).

The time evolution of this regolith migration during the spin-up process is shown in Fig. 1c. The regolith remains stable at first. When the progenitor reaches the critical spin period $T_{\mathrm{c}}=4.35 \mathrm{hr}$, a localized landslide first occurs at mid-latitudes ${ }^{9,21}$. Note that $T_{\mathrm{c}}$ here is for surface landslide failure, which is different from the shedding limits and internal failure limits ${ }^{7,8}$. The regolith mi- 
gration forms a narrow zone with intense shear strain (Fig. 1c, first panel). Within the shear band, particle contacts break and remake. Such rearrangement events soften the shear strength of the granular material $^{22,23}$, i.e., the particles in this zone rotate and slip, transporting the regolith above this weakened band downslope along the failure surface like a frozen ice block, with an average creeping speed of less than $1 \mathrm{~mm} / \mathrm{s}$ (Fig. 1b). Regolith above the slip region remains stable and undisturbed due to the low surface slope near the pole ${ }^{9}$. With increasing spin rate $\Omega$, the scarp at the head of the slip zone retreats towards high latitudes, leading to the shrinking of the stable region, as shown by the kinetic energy distribution change in Supplementary Fig. 3, in parallel with the massive mass wasting towards low latitudes. The retreat of the headscarp to high latitudes and the expansion of the depletion region at mid-latitudes are both evident from the growth of the failure surface (Fig. 1c, second panel). In the later stage of the migration, due to the existence of a stable region near the equator (low surface slope), the barely moved grains here increase the resisting force along the failure surface in the landslide foot, blocking the material in the head from moving towards the foot. Thus, the creeping debris moves uphill and then overlies the pre-existing materials, forming a distinct deposit bulge near the equator. The original shear band propagates to low latitudes and subsequently creates a rupture surface inside the deposition (Fig. 1c, third panel). It is notable that the elevated geologic upheaval that marks the foot of the landslides is not at the equator at first, i.e., the collapse is limited to the mid-latitudes due to the resistance force between regolith grains. Then, as the accumulation of downslope materials from the depletion zone goes on, this bulge swells upward, leading to a steeper local slope that eventually collapses towards the equator. The raised, pronounced equatorial ridge denotes a fully sped-up progenitor for which 
the top-shaped morphology emerges (Fig. 2a). The simulated topography, with low elevation at mid-latitudes, mean elevation at the polar region and high elevation at the equator, resembles the observed shapes of typical top-shaped asteroids (Fig. 2b), which is consistent with previous findings that regolith migrations are a major force that has reshaped these asteroids if the interior is stronger $^{7-9}$. Simulations with higher friction give a similar picture, except that the stable region near the pole is larger and that the equatorial ridge is steeper (Supplementary Video 6).

The motion of boulders across the surface shows three distinct dynamical behaviours (see Supplementary Videos $2-5$ ). The $15^{\circ}$ and $45^{\circ}$ boulders are located in the early migration zone, thus they start to creep downslope in synchronization with the first landslide of granular regolith. During the creeping-down process of the $45^{\circ}$ boulder, the strength of its supporting force chain network is weakened due to the grain agitations induced by the shear deformation ${ }^{24}$. These weak grain bonds generate a fluid-like behaviour; thus this boulder sinks into the regolith layer by a few metres and becomes tilted, reminiscent of the abundant partially buried boulders on Ryugu and Bennu ${ }^{12,13}$. This flow-induced submergence phenomenon has also been observed in granular experiments $^{22,24}$. At lower latitudes, the $15^{\circ}$ boulder is first elevated by the extruded underlying sediment, subsequently transferred downslope to the foot of the deposit upheaval, and eventually buried entirely by the accumulated debris from the collapsed bulge hill. This mechanism, i.e., boulders initially located at low latitudes being totally buried by the downslope deposition, may have caused the deficiency of large boulders at the equator as observed on Ryugu ${ }^{10}$ and Bennu ${ }^{25}$. At higher latitudes, the $75^{\circ}$ boulder remains static until the regressive headscarp of the depletion zone reaches $75^{\circ}$ latitude. The growth of this scarp removes support from adjacent grains, forming 
a fragile force-chain network downstream (Fig. 3). The weak support increases the equivalent local slope and thus triggers a downslope migration of the boulder. Finally, this boulder sinks into the granular mantle and leaves only a partially exposed body above the regolith layer, generating a distinct outcrop-like landform (Fig. 4). The $87^{\circ}$ boulder, however, is located in a stable region (see Fig. 2a) and thus does not move throughout the reshaping process of the top-shaped asteroids. This result is consistent with the geologic indication of a stable pole deduced from the disorganized boulder structure near Bennu's pole and the non-slipping polar boulder Otohime on Ryugu ${ }^{1,12}$. We infer that Otohime could be a primordial relic of the original disruption/reaccumulation of Ryugu's parent body. Future studies on the geologic age of Otohime from thermal or fracture properties would provide significant constraints on when the catastrophic impact event that formed Ryugu occurred. Note that we do not rule out the possibility that other exhumation processes could contribute to the subsequent evolution of these boulders. Some of the buried boulders in the equatorial region on Ryugu could be exhumed again by migrating grains from the ridge towards the current geopotential lows at higher latitudes ${ }^{12}$. Other mechanisms including Brazil nut effect ${ }^{20}$ and ballistic sorting ${ }^{19}$ could also further modify these landforms.

Our results show that the top shapes of rubble pile asteroids can be evolved via regolith migrations from mid-latitudes towards the equator driven by the YORP spin-up effect. If the interior is stronger than the outer regolith, then miniature surface landslides could be frequent as the spin rate approaches the critical spin limit ${ }^{9,21}$. These landslides are to be distinguished from their counterpart on large-gravity bodies like Mars, the Moon or Earth. On such planets the large boulders usually bounce and deposit near the toe of the avalanche sediment ${ }^{26}$. By contrast, regolith migra- 
tion on low gravity rubble piles, driven by YORP, once near the spin limit, creeps imperceptibly at glacial speeds. A detailed look into these creep-like landslides shows that the boulders are in a “co-evolving" relationship with the underlying granular regolith, which may account for the unique geological landforms observed on top-shaped asteroids, i.e., the stable boulders near the pole, the abundant partially buried boulders and the deficiency of large boulders at the equator. We find consistency of the derived shapes and boulder features between the observations and the numerical results, which lends support to our model as a way to decipher the evolution history of top-shaped asteroids. Further simulations show that the large craters that overlay the equator of Ryugu would have been eroded by the migrating regolith if they were formed before the equatorial ridges (Supplementary Fig. 7), implying an early-stage formation of the overall shapes ${ }^{1}$. This would appear to be in tension with the long timescales over which YORP effect could significantly modify the spins $^{27}$. A plausible explanation is that the progenitor bodies that emerged from the catastrophic disruptions are already oblate shapes with relatively high spin rates, which is a possible result of the direct reaccumulation of fragments in the case of large starting angular momentum ${ }^{27}$. These early-formed "diamond-shaped" rubble piles with high spin rates could serve as a midway starting point that facilitates the formation of top shapes, i.e., the YORP-induced migrations further reshaped the bodies and remodeled their surface features like large boulders to the current geomorphology. Another possibility is these craters could be created by detachment of a small chunk from the equatorial ridge at a more rapid rotation state ${ }^{28}$. This mechanism seems to be plausible given that the past spin period of Ryugu could be up to around 3 hours as deduced from its east-west dichotomy ${ }^{29}$. In such a scenario, the circum-equatorial ridge and overlying craters could both be 
landscapes sculpted by YORP spin-up.

\section{Methods}

We performed discrete element simulations ${ }^{14-16}$ using an original soft-particle $N$-body code DEMBody ${ }^{30}$, which is capable of tracing the quasi-static behaviour of particle-particle and particle-polyhedron interactions. In this code, the nonlinear contact forces between two contacting objects (spheresphere, sphere-polyhedron, sphere-wall) are calculated based on Hertz-Mindlin contact theory ${ }^{31}$ according to the mutual overlap (typically $<1 \%$ of their radii). A dimensionless coefficient $\mu$ is used to control the stick-slip friction between colliding particles. A physically based rotational resistance model incorporating rolling and twisting friction was implemented into this code, parameterized by a quantity $\beta$ that represents a statistical measure of real particle nonsphericity ${ }^{30,32}$. To calculate the contact between non-spherical boulders and grains, the boulder is represented as an assembly of movable walls with the same geometry and inertia tensor ${ }^{30}$, which allows it to behave as a rigid body. A second-order leapfrog integrator is used for integration of the equations of motion of both particles and boulders.

Our simulation scheme is as follows. First, we prepared a polydisperse granular medium consisting of 2,063,044 cohesionless particles with properties chosen according to Ryugu's regolith ${ }^{1}$, i.e., material density of $2.4 \mathrm{~g} / \mathrm{cc}$. A slight dispersion in the grain radius was considered, with a random distribution ranging from $0.4 \mathrm{~m}$ to $0.6 \mathrm{~m}$ to avoid any crystallization of the packing. The particles were dropped onto a rigid, frictional shell with a radius of $400 \mathrm{~m}$ and width of $100 \mathrm{~m}$ confined by two vertical frictionless walls ${ }^{9}$, as shown in Fig. 1a, forming a longitudinal slice of 
the asteroid surface with a thickness of $\sim 30 \mathrm{~m}$. A cuboid boulder, the size of which is similar to that of typical boulders on Ryugu, was initially located above the interface of the granular media. The whole system is under the two-component gravity ${ }^{9}$ exerted by the rocky interior (bulk density of $1.2 \mathrm{~g} / \mathrm{cc})^{1}$ and the regolith shell. After a settling-down phase of the granular-polyhedron system, we started to accelerate the asteroid linearly following the prescribed spin-up path shown in Fig. 1b. The acceleration was performed until massive debris shedding occurred at the equator. The angular acceleration used in this study is $2.5 \times 10^{-10} \mathrm{rad} / \mathrm{s}^{2}$, which is much smaller than the reference values used in previous works ${ }^{8,32}$. By using this slow spin-up acceleration, we confirm that the motion of the granular grains would immediately stop at any point in time when the spinup is stopped, which means that the regolith layer maintains quasi-static equilibrium during the spin-up process (see Supplementary Information).

Regolith bed geometry. We investigated the dependency of the boulder evolution on the geometry of the regolith bed. By using granular beds with depth of $45 \mathrm{~m}$ and of $20 \mathrm{~m}$, which consists of 3,246,524 particles and 1,452,565 particles, respectively, we tested the influence of regolith bed depth (Supplementary Figure 4-5). Simulations give results similar to those described above, in which tilted boulders and buried boulders emerge with the surface migration driven by YORP spin-up. By using a granular bed with width of $160 \mathrm{~m}$, which consists of 3,332,716 particles, we tested the influence of confining sidewalls (Supplementary Video 7). Simulations indicate the size of surrounding walls is large enough to eliminate the boundary effect. By using a spherical wedgeshaped bed with angular width of $20^{\circ}$, which consists of $2,063,045$ particles, we tested the influence of the boundary shape (Supplementary Figure 6). Simulations indicate the shrinkage effect from 
surrounding walls has only a minor influence on the boulder dynamics for our simulation settings. By using a granular bed of both hemispheres, which consists of 4,126,088 particles, we tested the symmetry of the regolith migration. Simulations show the sliding debris meet and accumulate symmetrically at the equator (Supplementary Video 9). The overall geomorphology is similar to that in hemisphere cases.

Equatorial crater. We investigated the evolution of equatorial craters during the YORP spin-up. A crater with radius of $80 \mathrm{~m}$ was generated at the equator before rotational acceleration (Supplementary Fig. 7). Simulations show that the migrating debris would have filled the equatorial craters if these craters formed before the equatorial ridges, which implies an early-stage formation of the overall morphology.

Boulders configuration. We investigated the dependency of the boulder evolution on their configurations. Simulations with boulders ten meters below the regolith surface show these initially underground boulders keep buried throughout the creeping evolution (Supplementary Videos 1011). We also tested the interaction between multiple boulders. Given that the number density of boulders ${ }^{10}$ larger than $15 \mathrm{~m}$ on Ryugu is $\sim 100 \mathrm{~km}^{-2}$, we randomly placed eight boulders, either on the surface or embedded in regolith, at middle and low latitudes of the simulated longitudinal slice. Simulations give results similar to those described above, in which surface boulders sink into or are buried by the creeping regolith, while underground boulders remain buried (Supplementary Video 12). 
Data availability. The data that support the plots within this paper and other findings of this study are available from the corresponding authors upon reasonable request.

Code availability. The code used to generate the datasets is available from the corresponding author on reasonable request.

1. Watanabe, S. et al. Hayabusa2 arrives at the carbonaceous asteroid 162173 Ryugu-A spinning top-shaped rubble pile. Science 364, 268-272 (2019).

2. Lauretta, D. S. et al. The unexpected surface of asteroid (101955) Bennu. Nature 568, 55-60 (2019).

3. Benner, L. A. et al. Radar imaging and a physical model of binary asteroid 65803 Didymos. In Bull. Am Astron. Soc., vol. 42, 1056 (2010).

4. Busch, M. W. et al. Radar observations and the shape of near-Earth asteroid 2008 EV5. Icarus 212, 649-660 (2011).

5. Ostro, S. J. et al. Radar imaging of binary near-Earth asteroid (66391) 1999 KW4. Science 314, 1276-1280 (2006).

6. Walsh, K. J., Richardson, D. C. \& Michel, P. Rotational breakup as the origin of small binary asteroids. Nature 454, 188-191 (2008).

7. Hirabayashi, M., Sánchez, P. \& Scheeres, D. J. Internal structure of asteroids having surface shedding due to rotational instability. Astrophys. J. 808, 63 (2015). 
8. Sánchez, P. \& Scheeres, D. J. Rotational evolution of self-gravitating aggregates with cores of variable strength. Planet. Space. Sci. 157, 39-47 (2018).

9. Sánchez, P. \& Scheeres, D. J. Cohesive regolith on fast rotating asteroids. Icarus 338, 113443 (2020).

10. Michikami, T. et al. Boulder size and shape distributions on asteroid Ryugu. Icarus 331, 179-191 (2019).

11. DellaGiustina, D. N. et al. Properties of rubble-pile asteroid (101955) Bennu from OSIRISREx imaging and thermal analysis. Nat. Astron. 3, 341-351 (2019).

12. Sugita, S. et al. The geomorphology, color, and thermal properties of Ryugu: Implications for parent-body processes. Science 364, 252 (2019).

13. Walsh, K. J. et al. Craters, boulders and regolith of (101955) Bennu indicative of an old and dynamic surface. Nat. Geosci. 12, 242-246 (2019).

14. Cundall, P. A. \& Strack, O. D. A discrete numerical model for granular assemblies. Geotechnique 29, 47-65 (1979).

15. Sánchez, P. \& Scheeres, D. J. Simulating asteroid rubble piles with a self-gravitating softsphere distinct element method model. Astrophys. J. 727, 120 (2011).

16. Schwartz, S. R., Richardson, D. C. \& Michel, P. An implementation of the soft-sphere discrete element method in a high-performance parallel gravity tree-code. Granul. Matter 14, 363-380 (2012). 
17. Barnouin, O. S. et al. Shape of (101955) Bennu indicative of a rubble pile with internal stiffness. Nat. Geosci. 12, 247-252 (2019).

18. Arakawa, M. et al. An artificial impact on the asteroid (162173) Ryugu formed a crater in the gravity-dominated regime. Science 368, 67-71 (2020).

19. Wright, E. et al. Boulder stranding in ejecta launched by an impact generated seismic pulse. Icarus 337, 113424 (2020).

20. Matsumura, S. et al. The brazil nut effect and its application to asteroids. Mon. Not. R. Astron. Soc. 443, 3368-3380 (2014).

21. Scheeres, D. J. et al. The dynamic geophysical environment of (101955) Bennu based on OSIRIS-REx measurements. Nat. Astron. 3, 352-361 (2019).

22. Reddy, K., Forterre, Y. \& Pouliquen, O. Evidence of mechanically activated processes in slow granular flows. Phys. Rev. Lett. 106, 108301 (2011).

23. Germanovich, L. N., Kim, S. \& Puzrin, A. M. Dynamic growth of slip surfaces in catastrophic landslides. Proc. Math. Phys. Eng. Sci. 472, 20150758 (2016).

24. Nichol, K., Zanin, A., Bastien, R., Wandersman, E. \& van Hecke, M. Flow-induced agitations create a granular fluid. Phys. Rev. Lett. 104, 078302 (2010).

25. Walsh, K. J. et al. Geology of Bennu's equatorial ridge. In Asteroid Science 2019 (2019).

26. Gray, J. M. N. T. Particle segregation in dense granular flows. Annu. Rev. Fluid. Mech. 50, 407-433 (2018). 
27. Michel, P. et al. Collisional formation of top-shaped asteroids and implications for the origins of Ryugu and Bennu. Nat. Commun. 11, 1-11 (2020).

28. Tardivel, S., Sánchez, P. \& Scheeres, D. J. Equatorial cavities on asteroids, an evidence of fission events. Icarus 304, 192-208 (2018).

29. Hirabayashi, M. et al. The western bulge of 162173 Ryugu formed as a result of a rotationally driven deformation process. Astrophys. J. Lett. 874, L10 (2019).

30. Cheng, B., Yu, Y. \& Baoyin, H. Numerical simulations of the controlled motion of a hopping asteroid lander on the regolith surface. Mon. Not. R. Astron. Soc. 485, 3088-3096 (2019).

31. Somfai, E., Roux, J.-N., Snoeijer, J. H., Van Hecke, M. \& Van Saarloos, W. Elastic wave propagation in confined granular systems. Phys. Rev. E. 72, 021301 (2005).

32. Zhang, Y. et al. Rotational failure of rubble-pile bodies: Influences of shear and cohesive strengths. Astrophys. J. 857, 15 (2018).

33. Peters, J. F., Muthuswamy, M., Wibowo, J. \& Tordesillas, A. Characterization of force chains in granular material. Phys. Rev. E. 72, 041307 (2005).

34. Hayabusa2 Optical Navigation Camera (ONC) dataset at JAXA Data Archives and Transmission System (DARTS), http://darts.isas.jaxa.jp/pub/hayabusa2/onc_bundle

Corresponding Author Correspondence and inquiries for materials should be addressed to H.B. and Y.Y. 
Acknowledgements This work is funded by the National Science Fund for Distinguished Young Scholars of China (No. 11525208). Y.Y. acknowledges support from the Natural Science Foundation of China (No. 11702009). P.M. acknowledges funding from the French space agency CNES, from the European Union's Horizon 2020 research and innovation programme under grant agreement No. 870377 (project NEO-MAPP) and from Academies of Excellence: Complex systems and Space, environment, risk, and resilience, part of the IDEX JEDI of the Université Côte d'Azur. The authors acknowledge supports from JSPS Core-to-Core program "International Network of Planetary Sciences". We are grateful to the Hayabusa2 ONC team for providing images which are available at the JAXA Data Archives and Transmission System (DARTS) at http://darts.isas.jaxa.jp/pub/hayabusa2/onc_bundle.

Author contributions $\quad$ B.C. performed the soft-sphere numerical simulations and analysed the numerical results. B.H. and Y.Y. initiated the project, designed the simulations and led the research. E.A. and D.C.R contributed to the discussion of the two layer model and the creeping process. M.H. provides essential comments on the interior structure and deformation. P.M. initiated the collaboration between the institutions with Y.Y. and provided outstanding questions on scope of the research with M.Y. All authors contributed to interpretation of the results and preparation of the manuscript.

Competing Interests The authors declare no competing interests. 
298 List of Figures 
Figure 1 Asteroid morphological change during the YORP spin-up process. a, "Two-layer structure" with a shallow mantle of loose granular regolith covering a stiffer interior. A large boulder lies on the regolith surface. b, Spin-up path and consequent creep speed of global regolith migrations for a $75^{\circ}$ boulder with moderate-friction regolith case. c, Four selected frames of the regolith and boulder at different spin periods showing the regolith migration under the YORP effect. The cuboid represents the boulder and is located at $75^{\circ}$ latitude initially. The regolith has moderate friction. When the progenitor reaches the critical spin period $T_{\mathrm{c}}=4.35 \mathrm{hr}$, the centrifugal tension induces a narrow zone with intense shear strain (dashed line in first panel; see Supplementary Fig. 2 for the shear heat of regolith particles). This shear band expands towards high latitudes, and creeping debris moves to low latitudes along the rupture surface in the deposition (dashed line in third panel). At the end of the simulation, deposit sediment accumulates at low-latitudes, forming a pronounced ridge along the equator (fourth panel). The failure surface acts as a dynamical divider separating the static zone from the depletion zone, whose growth controls the landslides across the regolith bed. Animation of this figure: Supplementary Video 1. 
Figure 2 The simulated geomorphology. a, The topography changes during the YORP spin-up process, showing the barely moved pole and migrating debris from midlatitudes overlying the pre-existing materials near the equator. Here the elevation is the radial height of particles above the interior shell. Data are for a moderate-friction case. b, Simulated morphological profile (black line) compared with Ryugu's (blue) ${ }^{1}$, Bennu's (red) ${ }^{21}, 1999$ KW4's (green) ${ }^{5}$ and 2008 EV5's (yellow) ${ }^{4}$ average shapes. All profiles are normalized by the corresponding equivalent radius. Shaded areas stand for the $1 \sigma$ error bands. 
Figure 3 Visualization of the force networks excited by the creeping boulder. Colour indicates the elastic energy, $E$, stored in each particle relative to the average elastic energy, $E_{0}$. Note that only those particles with $E>E_{0}$ are considered part of the force chains $^{33}$. The white-curve indicates the free surface of the creeping regolith. Red arrows show the sinking and tilting directions of the boulder. The chain structure downstream is significantly fragmented and spatially sparse due to the removal of adjacent grains by the retrogressive landslide, leading to subduction migration of the boulder. Data are for a $75^{\circ}$ boulder with moderate-friction regolith case. 
Figure 4 Typical landforms of boulders sculpted by YORP evolution. The $87^{\circ}$ boulder remains stable, consistent with the non-slipping polar boulder Otohime on Ryugu ${ }^{12}$. The boulders at mid-latitudes sink into the regolith layer by a few metres and become tilted. These landforms generated by regolith migrations are likely the geophysical origins of the partially buried/oblique boulders on Ryugu ${ }^{12}$ and Bennu ${ }^{13}$. (panel d, hyb2_onc_20180831_101059 panel e, hyb2_onc_20180801_160642_tnf_l2c and panel f, hyb2_onc_20180720_075208_tvf_l2b are from Hayabusa2 ONC dataset ${ }^{34}$.) 

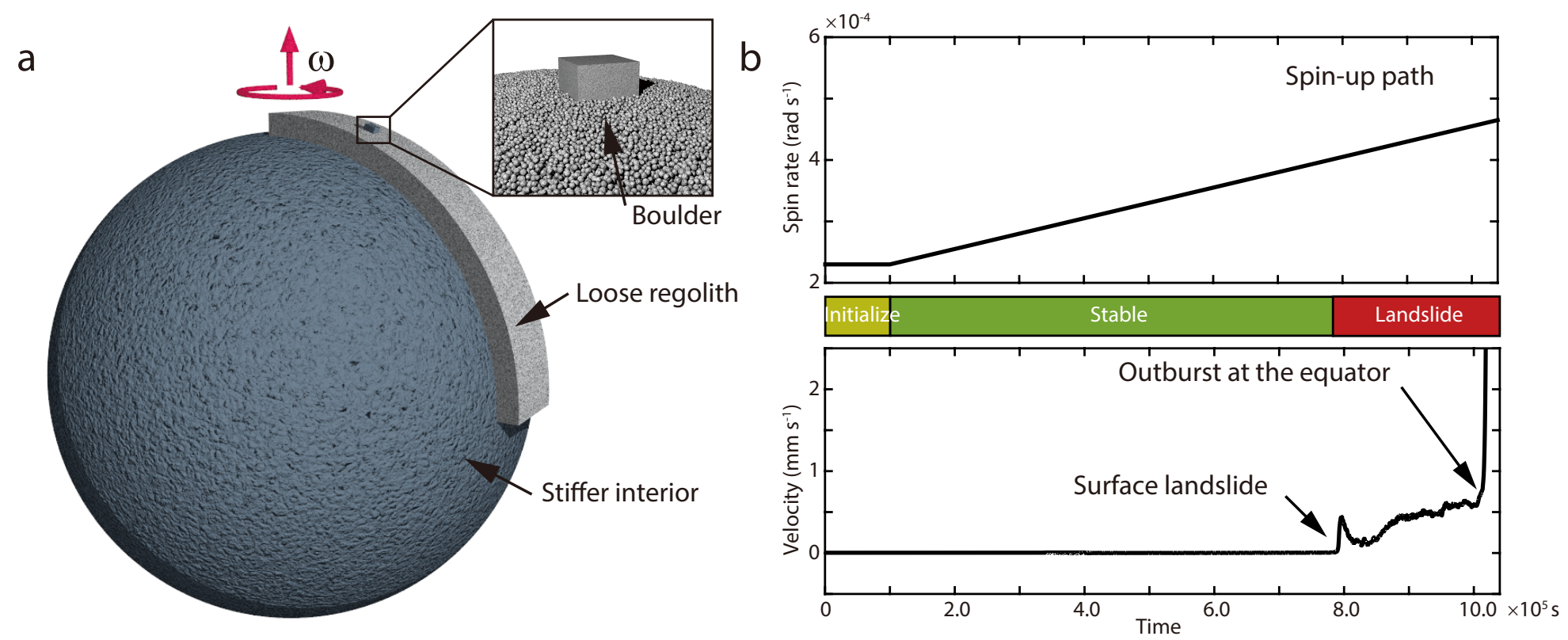

C

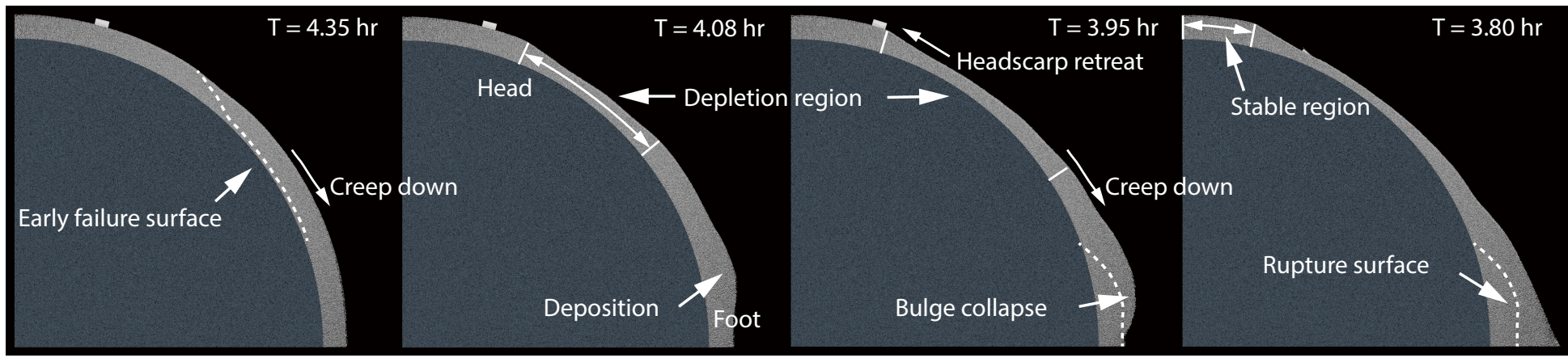









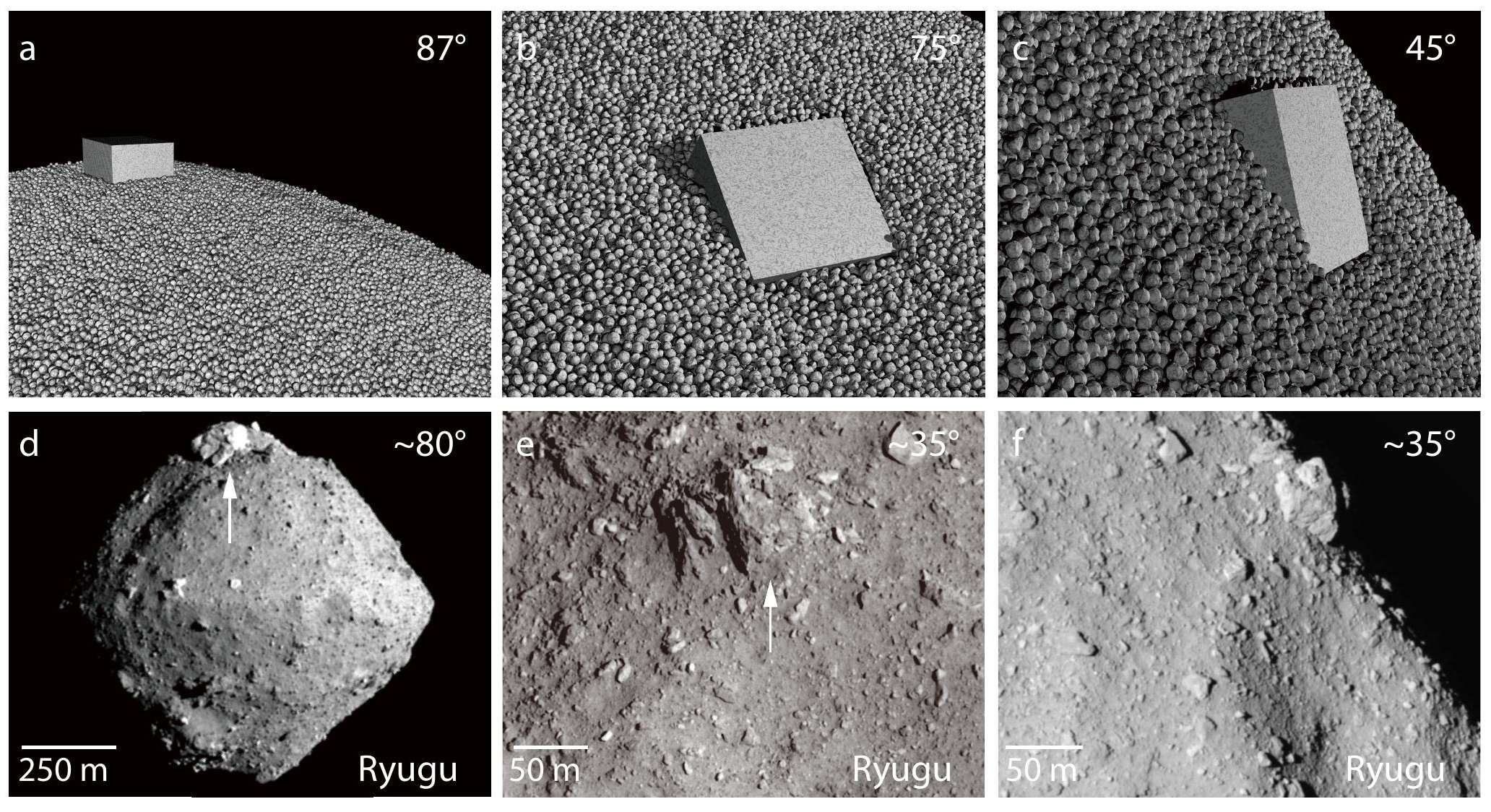

